\title{
Dense and hot matter in compact stars and heavy-ion collisions
}

\author{
Stefan Schramm ${ }^{1, \star}$, Veronica Dexheimer ${ }^{2}$, Ayon Mukherjee $^{1}$, and Jan Steinheimer ${ }^{1}$ \\ ${ }^{1}$ FIAS, Ruth-Moufang-Str. 1, D-60438 Frankfurt am Main, Germany \\ ${ }^{2}$ Department of Physics, Kent State University, Kent, OH 44242, USA
}

\begin{abstract}
We discuss the effect of exotic particles in neutron star matter and the corresponding impact on gross properties of neutron stars within effective models for the strong interaction. Particularly, for the quark-hadron parity-doublet model, we show results for compact star properties and discuss the phase structure of the model and its possible relevance for heavy-ion collision phenomenology.
\end{abstract}

\section{Introduction}

Studying the phase structure of hot and dense strongly interacting matter is central to many experimental and theoretical efforts in modern nuclear physics. As a complement to a range of ultrarelativistic heavy-ion collision experiments targeting in particular hot matter, the environment of neutron stars samples high-density matter at very low temperature. In general, one cannot observe the dense matter directly, but the observations of stellar properties like mass, radius and cooling behaviour have to be interpreted using theoretical models in order to constrain the equation of state of stellar matter. In order to calculate strong interaction matter relevant for compact stars as well as heavy-ion physics we have developed and studied hadronic and hadron-quark models within the so-called chiral mean field (CMF) approach.

\section{Modeling compact stars}

A realistic hadronic model of dense matter as it exists in neutron stars has to contain, in addition to nucleons, hyperons as degrees of freedom. One example is the hadronic chiral mean field model [1], where baryons and mesons from the lowest SU(3) multiplets are coupled linearly:

$$
\mathcal{L}_{\mathrm{B}}=-\sum_{i} \bar{\psi}_{i}\left[\gamma_{0}\left(g_{i \omega} \omega+g_{i \phi} \phi+g_{i \rho} \rho\right)+g_{i \sigma} \sigma+g_{i \delta} \delta+g_{i \zeta} \zeta+M_{0_{i}}\right] \psi_{i}
$$

The baryon fields $\psi_{i}$ interact with the mean fields of the vector mesons (the isoscalar $\omega$, the isovector $\rho$, and the isoscalar field with hidden strangeness $\phi$ ), as well as with the corresponding scalar fields $\sigma, \delta$, and $\zeta . M_{0_{i}}$ is a small explicit mass term. The coupling strengths $g_{B M}$ follow from a fitting of vacuum properties like baryon masses, nuclear matter properties and hypernuclear binding energies, as discussed in $[1,2]$.

^e-mail: schramm@ fias.uni-frankfurt.de 
However, independent of the interaction model, including hyperons (or other states) as additional degrees of freedom softens the equation of state and in turn reduces the maximum star mass. Therefore, there has to be a mechanism that suppresses (or removes) the amount of hyperons at densities relevant for compact star physics. One possibility is the vector repulsion of hyperons generated by the exchange of the $\phi$ meson [3-5]. This finding reinforces the point that when including hyperons as parts of the baryon octet, one should also take into account the corresponding SU(3) multiplets in the meson sector. As illustration of the effect of the $\phi$ exchange, Fig. 1 shows the maximum stellar mass that can be reached by solving the Tolman-Oppenheimer-Volkov (TOV) equations $[6,7]$ for a static star using the equation of state from the CMF model including hyperons. The curve illustrates the effect on the stellar mass when the value of the baryon- $\phi$ meson coupling is decreased down to 0 . One can clearly observe the substantial reduction of the stellar mass if one neglects the effects of the $\phi$ meson.

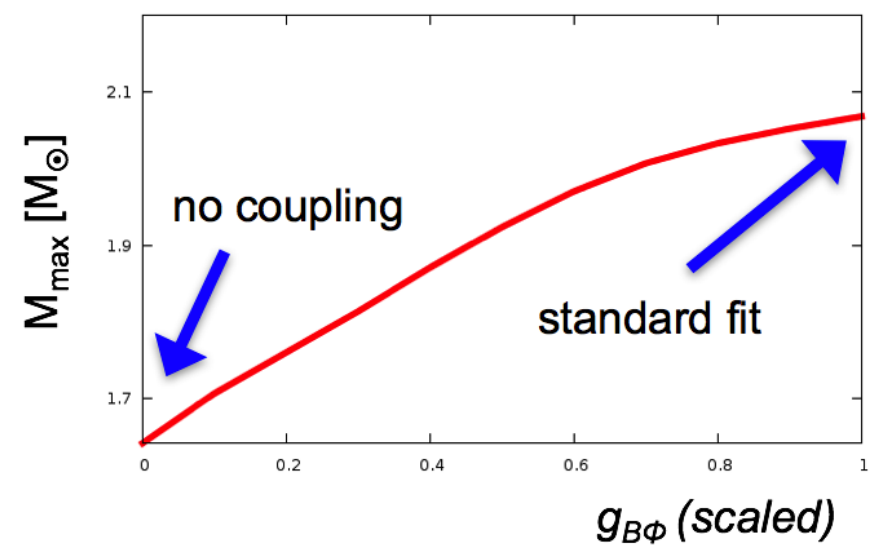

Figure 1. Maximum stellar mass as function of the normalized baryon- $\phi$ meson coupling strength calculated within the CMF model.

Another possibility to avoid the softening of the equation of state is the change of degrees of freedom through a transition to quark matter. However, in studies using a hadronic model combined with a simple MIT-bag quark model [8] or in combined quark-hadron approaches based on the CMF model [9], a big latent energy is found in the phase transition to quarks. This leads to a softening of the equation of state and, for very strong phase transitions, stars with a quark core are not stable static solutions of the TOV equations. This is a general behaviour for a strong phase transition that can significantly reduce maximum star masses [10]. This effect on the masses might not necessarily be too drastic, however, depending on the transition point. Fig. 2 shows the stellar mass in the purely hadronic CMF model as function of the stellar central density. Here, the maximum mass of about 2.1 solar masses is reached at a density $\rho_{\max } \approx 6 \rho_{0}$. The function is rather flat around this value. Consequently, in case exotic components like quarks or other particles appear at some lower density, creating a large latent energy and an instability of the compact star, the maximum mass would still not be reduced significantly, even if this density is, say, a third lower than $\rho_{\max }$ due to the flatness of the curve, this is a rather model-independent feature.

Alternatively, a combined quark-hadron approach based on the CMF model in the so-called paritydoublet formulation has been developed $(\mathrm{Q} \chi \mathrm{P}$ model $)[11,12]$. The main feature of such a model is that the octet of baryons us combined with their opposite parity partners [13-16], while quark degrees 


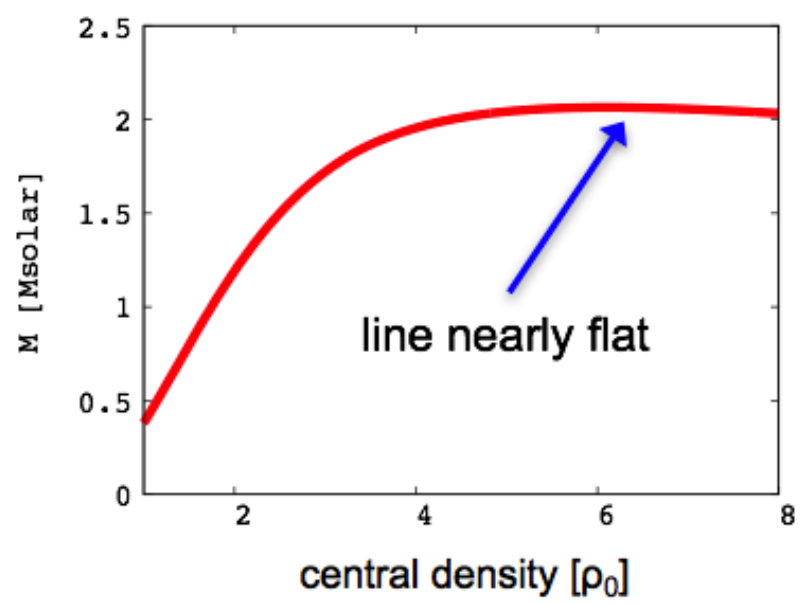

Figure 2. Stellar mass of the hadronic star, calculated within the CMF model, as function of the central density in units of nuclear saturation density.

of freedom that couple linearly to the mean fields as in Eq. 1 are introduced. In the case of the nucleon, this state might be the $\mathrm{N}(1535)$ resonance. For the hyperons the situation is not completely clear, here, for simplicity, we assume equal vacuum mass splitting of the various parity partners [13]. Note that related model studies for isospin-symmetric matter have been performed in a 2-flavor quark-mesonnucleon approach $[17,18]$.

In the parity-doublet approach the baryonic effective masses read

$$
m_{\mathrm{i} \pm}^{*}=\sqrt{\left[\left(g_{\sigma \mathrm{i}}^{(1)} \sigma+g_{\zeta \mathrm{i}}^{(1)} \zeta\right)^{2}+\left(m_{0}+n_{\mathrm{s}} m_{\mathrm{s}}\right)^{2}\right]} \pm g_{\sigma \mathrm{i}}^{(2)} \sigma \pm g_{\zeta \mathrm{i}}^{(2)} \zeta .
$$

There are two possible coupling terms of the scalar fields and baryons $g^{(1)}, g^{(2)}$, where the second one generates the splitting of the parity partners. Here, the term $n_{\mathrm{s}} m_{\mathrm{S}}$ is an explicit flavor-breaking term, where $n_{\mathrm{S}}$ is the number of strange quarks in the baryon, and $m_{0}$ is a chirally invariant mass term. Note, that the mass of the doublets (in the equation denoted by the \pm sign) is degenerate for vanishing scalar fields, similar to the degeneracy of the pion and sigma meson in the chirally restored phase of the linear sigma model. Using the equation of state resulting from this model, one obtains compact star masses and radii as shown in Fig. 3. The maximum mass is about two solar masses in agreement with accurately measured heavy pulsars $[19,20]$. As a quite unique feature of this approach, the calculated stars contain an extensive mixed phase of baryons and quarks [12], where the amount of quark matter for specific stars is indicated in the figure. Here, in contrast to the previous discussion of hybrid stars (neutron stars with a quark core), the mixed phase of quarks with baryons that have a strong repulsive interaction is stiff enough to support stable heavy compact stars. Note the rather small values for the stellar radius in agreement with various studies of low-mass X-ray binary systems [21, 22].

\section{Hot and dense matter}

In calculating the phase structure of dense and hot matter within the $\mathrm{Q} \chi \mathrm{P}$ model, one obtains the phase diagram shown in Fig. 4. The bands show the transition region of the scalar condensate and 


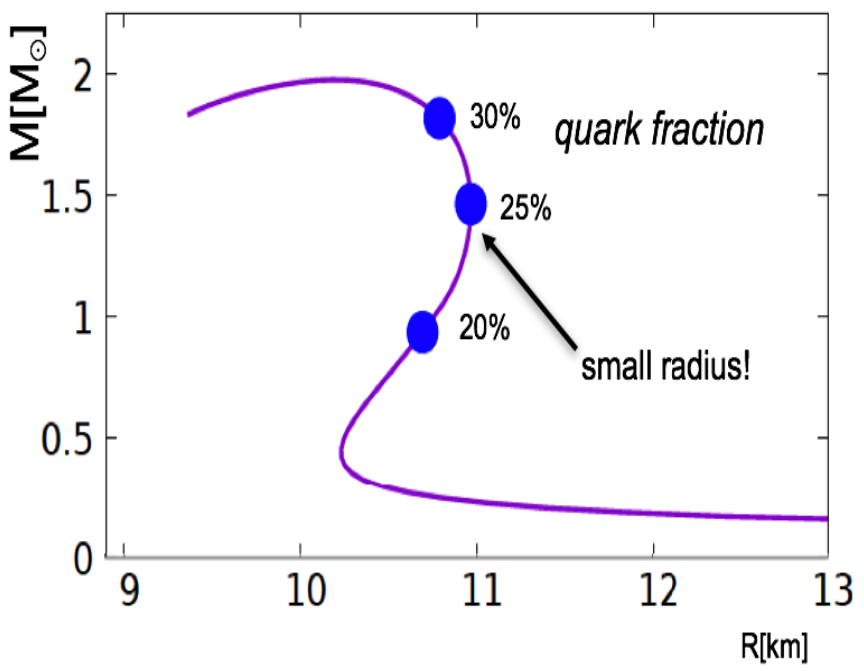

Figure 3. Mass-radius diagram of hybrid stars in the $\mathrm{Q} \chi \mathrm{P}$ model. The circles indicate different amounts of quark matter in the star.

the Polyakov loop field $\Phi$ that effectively describes the effect of the confinement as discussed in Refs. $[9,11,23]$. The full lines indicate first-order phase transitions. There are two first-order transitions: the one at lower chemical potential is the liquid-gas transition, whereas the second one corresponds to the deconfinement transition with a low critical end point temperature of about $20 \mathrm{MeV}$. While the first-order phase transitions vanish fast with rising temperature, these transitions, nevertheless, can have significant impact on heavy-ion physics phenomenology. This can be seen by calculating the baryon number susceptibilities of order $n, \chi_{n}^{B}$, defined as

$$
\frac{\chi_{\mathrm{n}}^{\mathrm{B}}}{T^{4-n}}=\frac{\partial^{\mathrm{n}}\left(P\left(T, \mu_{\mathrm{B}}\right) / T^{4}\right)}{\partial\left(\mu_{\mathrm{B}} / T\right)^{\mathrm{n}}}
$$

with $P$ denoting the pressure of the system. The susceptibilities are closely connected to particle number fluctuations that can be measured in heavy-ion experiments. Fig. 5 shows the ratio of the fourth- to second-order susceptibility. The black dashed lines indicate the continuation of the firstorder transition as crossover transitions. In addition, freeze-out curves [24] for different temperatures are indicated in the plot. As a very interesting feature of this figure, one can observe a strong effect, when the deconfinement and liquid-gas transition merge at high temperatures and low baryochemical potential. Thus, experimental signals are expected to be strongly affected by the interference of those two transitions (see Ref. [25]). Due to the treatment of the matter as homogeneous in this mean-field approach, nucleonic clustering effects have not been considered here. Therefore, an extension of this work along the lines considered in Refs. [26, 27], including clusters as effective degrees of freedom, should be pursued in future work.

In future studies, this approach will be applied to dynamical collision studies by implementing the equation of state in simulation codes for ultrarelativistic heavy-ion collisions. Work along this line is already in progress. 


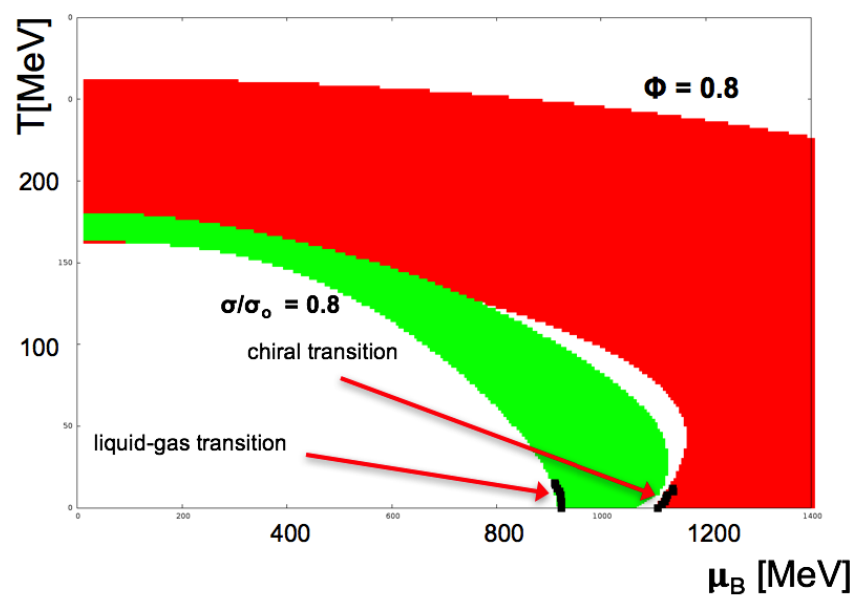

Figure 4. Phase structure of the $\mathrm{Q} \chi \mathrm{P}$ model in the $\mu_{B}-T$ plane for isospin symmetric matter with zero net strangeness. The filled areas indicate the range of the scalar condensate with $20 \%$ to $80 \%$ of the vacuum value, and the Polyakov loop field with values between 0.2 and 0.8 .

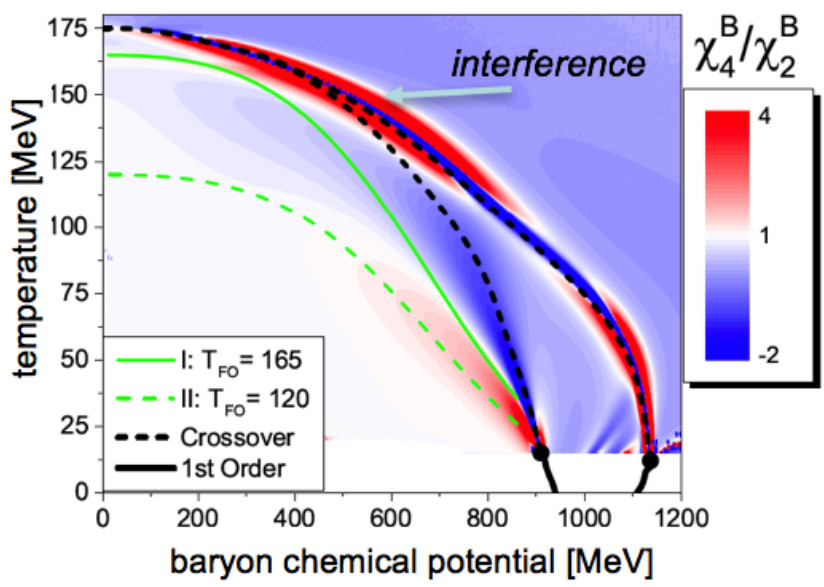

Figure 5. Ratio of baryon number susceptibilities $\chi_{4}^{B} / \chi_{2}^{B}$ for heavy-ion matter as function of chemical potential and temperature. Freeze-out curves for different parameters are indicated.

\section{Acknowledgements}

The authors acknowledge support from NewCompStar COST Action MP1304, HIC for FAIR, and from BMBF. Computational resources were provided by the Frankfurt Center for Scientific Computing (CSC). The authors thank the referee for pointing out the potential relevance of nuclear clusters in the interference of the phase transitions. 


\section{References}

[1] P. Papazoglou, D. Zschiesche, S. Schramm, J. Schaffner-Bielich, H. Stoecker, W. Greiner, Phys. Rev. C59, 411 (1999), nucl-th/9806087

[2] V. Dexheimer, R. Negreiros, S. Schramm, Phys. Rev. C91, 055808 (2015), 1411.4623

[3] V. Dexheimer, S. Schramm, Astrophys. J. 683, 943 (2008), 0802 . 1999

[4] I. Bednarek, R. Manka, J. Phys. G31, 1009 (2005), hep-ph/0506059

[5] J. Schaffner-Bielich, A. Gal, Phys. Rev. C62, 034311 (2000), nucl-th/0005060

[6] J.R. Oppenheimer, G.M. Volkoff, Phys. Rev. 55, 374 (1939)

[7] R.C. Tolman, Phys. Rev. 55, 364 (1939)

[8] R. Negreiros, V.A. Dexheimer, S. Schramm, Phys. Rev. C85, 035805 (2012), 1011.2233

[9] V.A. Dexheimer, S. Schramm, Phys. Rev. C81, 045201 (2010), 0901. 1748

[10] M.G. Alford, S. Han, Eur. Phys. J. A52, 62 (2016), 1508 . 01261

[11] A. Mukherjee, J. Steinheimer, S. Schramm, Phys. Rev. C96, 025205 (2017), 1611. 10144

[12] A. Mukherjee, S. Schramm, J. Steinheimer, V. Dexheimer (2017), 1706.09191

[13] J. Steinheimer, S. Schramm, H. Stocker, Phys. Rev. C84, 045208 (2011), 1108.2596

[14] Y. Nemoto, D. Jido, M. Oka, A. Hosaka, Phys. Rev. D57, 4124 (1998), hep-ph/9710445

[15] C. DeTar, T. Kunihiro, Phys. Rev. D 39, 2805 (1989)

[16] T. Hatsuda, M. Prakash, Phys. Lett. B224, 11 (1989)

[17] M. Marczenko, C. Sasaki, ArXiv e-prints (2017), 1711.05521

[18] S. Benić, I. Mishustin, C. Sasaki, Phys. Rev. D 91, 125034 (2015)

[19] P. Demorest, T. Pennucci, S. Ransom, M. Roberts, J. Hessels, Nature 467, 1081 (2010), 1010.5788

[20] J. Antoniadis et al., Science 340, 6131 (2013), 1304 . 6875

[21] S. Guillot, R.E. Rutledge, E.F. Brown, Astrophys. J. 732, 88 (2011), 1007.2415

[22] S. Guillot, M. Servillat, N.A. Webb, R.E. Rutledge, Astrophys. J. 772, 7 (2013), 1302.0023

[23] J. Steinheimer, S. Schramm, H. Stocker, J. Phys. G38, 035001 (2011), 1009. 5239

[24] A. Andronic, P. Braun-Munzinger, J. Stachel, Phys. Lett. B673, 142 (2009), [Erratum: Phys. Lett.B678,516(2009)], 0812.1186

[25] A. Andronic, P. Braun-Munzinger, K. Redlich, J. Stachel, ArXiv e-prints (2017), 1710.09425

[26] S. Typel, H.H. Wolter, G. Röpke, D. Blaschke, The European Physical Journal A 50, 17 (2014)

[27] N.U. Bastian, P. Batyuk, D. Blaschke, P. Danielewicz, Y.B. Ivanov, I. Karpenko, G. Röpke, O. Rogachevsky, H.H. Wolter, The European Physical Journal A 52, 244 (2016) 